Claremont Colleges

Scholarship@Claremont

WM Keck Science Faculty Papers

W.M. Keck Science Department

9-25-1995

\title{
Disorder and Synchronization in a Josephson Junction Plaquette
}

Adam S. Landsberg

Claremont McKenna College; Pitzer College; Scripps College

Yuri Braiman

Kurt Wiesenfeld

Georgia Institute of Technology

\section{Recommended Citation}

Landsberg, A.S., Y.Braiman, and K. Wiesenfeld. "Disorder and Synchronization in a Josephson Junction Plaquette." Applied Physics Letters 67.13 (1995): 1935-1937. DOI:10.1063/1.114573

This Article is brought to you for free and open access by the W.M. Keck Science Department at Scholarship @ Claremont. It has been accepted for inclusion in WM Keck Science Faculty Papers by an authorized administrator of Scholarship @ Claremont. For more information, please contact scholarship@cuc.claremont.edu. 


\title{
Disorder and synchronization in a Josephson junction plaquette
}

\author{
A. S. Landsberg, ${ }^{\text {a) }}$ Y. Braiman, and K. Wiesenfeld \\ School of Physics, Georgia Institute of Technology, Atlanta, Georgia 30332
}

(Received 24 April 1995; accepted for publication 24 July 1995)

\begin{abstract}
We describe the effects of disorder on the coherence properties of a $2 \times 2$ array of Josephson junctions (a "plaquette"). The disorder is introduced through variations in the junction characteristics. We show that the array will remain one-to-one frequency locked despite large amounts of the disorder, and determine analytically the maximum disorder that can be tolerated before a transition to a desynchronized state occurs. Connections with larger $N \times M$ arrays are also drawn. (C) 1995 American Institute of Physics.
\end{abstract}

Josephson junctions have potential importance for device applications, where one goal is to generate high-power, high-frequency voltage oscillations with a narrow linewidth. ${ }^{1,2}$ A single Josephson junction can convert a dc bias current into voltage oscillations as fast as a terahertz, ${ }^{3}$ an extraordinarily high frequency for an electronic device; however, the power output is rather small (typically about 10 $\mathrm{nW})$. Series arrays of $N$ identical junctions can boost power, ${ }^{1,4,5}$ though the coherence properties of such arrays are sensitive to disorder (i.e., variations in the junction parameters), which is inescapable in the fabrication of arrays. ${ }^{5-7}$ To overcome this difficulty, two-dimensional arrays have been proposed..$^{8-10}$ Experiments on $10 \times 10$ arrays by Benz and Burroughs $^{7}$ have yielded powers as high as $0.4 \mu \mathrm{W}$ at 150 $\mathrm{GHz}$, and numerical simulations by Octavio and co-workers ${ }^{6}$ on $8 \times 8$ arrays have revealed that although two-dimensional arrays can also be sensitive to disorder, they are less so than their one-dimensional counterparts.

At present though, a general theoretical understanding of the effects of disorder on the coherence properties of twodimensional arrays poses a significant challenge. Our strategy here is to study in detail disorder in the simplest of two-dimensional arrays, the $2 \times 2$ "plaquette," depicted in Fig. 1. The individual junctions are described by the RSJ model. The resistances of the junctions are identical, but their critical currents are randomly distributed. A constant current is fed in uniformly along the top row of superconducting sites, and removed uniformly along the bottom. The dynamics of the array can be described in terms of the phase differences $\left(\Phi_{T}, \Phi_{B}, \Phi_{L}, \Phi_{R}\right)$ across the superconducting sites. The equations of motion are

$$
\begin{aligned}
& \partial_{t} \Phi_{T}+I_{T} \sin \left(\Phi_{T}\right)=\Delta, \\
& \partial_{t} \Phi_{B}+I_{B} \sin \left(\Phi_{B}\right)=-\Delta, \\
& \partial_{t} \Phi_{L}+I_{L} \sin \left(\Phi_{L}\right)=I-\Delta, \\
& \partial_{t} \Phi_{R}+I_{R} \sin \left(\Phi_{R}\right)=I+\Delta,
\end{aligned}
$$

where $I$ denotes the external current fed into the system, and $\Delta$ (respectively $-\Delta$ ) is the spontaneously induced shunt current across the top (respectively bottom) junction. The shunt current $\Delta$ is determined by the supplemental constraint relation $\Phi_{T}+\Phi_{R}-\Phi_{B}-\Phi_{L}=0$, indicating that the sum of the

\footnotetext{
${ }^{a)}$ Electronic mail: ph287al@prism.gatech.edu
}

phase differences around a closed loop must vanish in the absence of any magnetic field. From this and Eqs. (1a)-(1d) it follows that

$$
\begin{aligned}
\Delta= & \frac{1}{4}\left[I_{T} \sin \left(\Phi_{T}\right)+I_{R} \sin \left(\Phi_{R}\right)-I_{B} \sin \left(\Phi_{B}\right)\right. \\
& \left.-I_{L} \sin \left(\Phi_{L}\right)\right] .
\end{aligned}
$$

Moreover, owing to the constraint, only three of the four phase differences are truly independent, so that (1a)-(1d) effectively reduces to a set of three coupled equations. In the idealized case of identical junctions $\left(I_{T}=I_{R}=I_{B}=I_{L}\right)$, it has been shown in Ref. 9 that the system is attracted to an "inphase" solution, in which the two vertical junctions are phase locked $\left[\Phi_{L}(t)=\Phi_{R}(t)\right]$, and the horizontal junctions are inactive $\left[\Phi_{T}(t)=\Phi_{B}(t)=0\right]$ (the transverse shunt current $\Delta$ being zero).

We have studied Eqs. (1a)-(1d) with disorder using a multiple time scale expansion. The asymptotic analysis assumes the plaquette is driven at high bias current ( $I$ $\left.\gg I_{L}, I_{R}, I_{T}, I_{B}\right)$, so that the vertical junctions are overturning rapidly. (In practice this condition can be relaxed significantly, as will be described later.) It is equivalent but more useful to treat the bias current as an (1) quantity and instead let the critical currents of the junctions be small $(\ll 1)$. Thus, defining $\epsilon$ to be a small parameter, we $\operatorname{set}^{11} I_{L}$ $-\epsilon I_{L}, I_{R} \rightarrow \epsilon I_{R}, I_{T} \rightarrow \epsilon^{2} I_{T}, I_{B} \rightarrow \epsilon^{2} I_{B}$ in Eqs. (1a)-(1d). For the multiple scale analysis, we define $t_{0} \equiv t, t_{1} \equiv \epsilon t, t_{2} \equiv \epsilon^{2} t$ to be fast, slow, and superslow time scales, respectively, and let $\partial_{t}=\partial_{t_{0}}+\epsilon \partial_{t_{1}}+\epsilon^{2} \partial_{t_{2}}$. Lastly, we expand the dynamical phases in (1a)-(1d) in powers of $\epsilon$ (e.g., $\Phi_{T}=\Phi_{T, 0}+\epsilon \Phi_{T, 1}$ $+\epsilon^{2} \Phi_{T, 2}$, etc. $)$.

Substituting the preceding expansions into Eqs. (1a)(1d), a hierarchy of equations is constructed based on powers of $\epsilon$. At each order, one solves the resulting equations with the help of auxiliary constraints known as "nonresonance conditions," which ensure that the asymptotic expansion remains uniformly valid. (See, e.g., Ref. 12 for a general description of this method.) In the absence of disorder, we recover the stable, inphase solution of Ref. 9. For weak disorder, we find that the system is attracted to a frequencylocked state, in which the two vertical junctions overturn at the same average rate $\left(\left\langle\dot{\Phi}_{L}\right\rangle=\left\langle\dot{\Phi}_{R}\right\rangle \neq 0\right)$. Close to the identical-junction limit, this frequency-locked solution is nearly in phase, but the phase shift increases with the disorder. The horizontal junctions meanwhile are active, but do not overturn $\left(\left\langle\dot{\Phi}_{T}\right\rangle=\left\langle\dot{\Phi}_{B}\right\rangle=0\right)$, indicating that they do not 


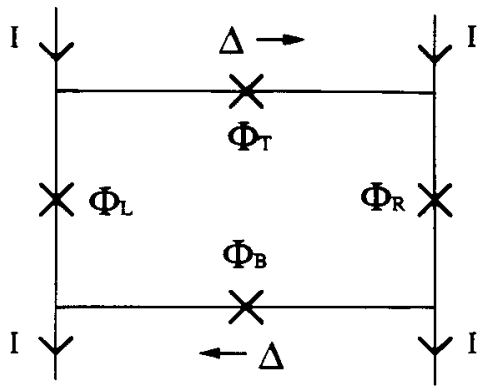

FIG. 1. The Plaquette. The phase differences across the vertical and horizontal junctions are denoted by $\Phi_{L}, \Phi_{R}$ and $\Phi_{T}, \Phi_{B}$, respectively. Current $I$ is imposed at the top and removed at the bottom; $\Delta$ is the induced current shunted across the horizontal junctions.

pass any regular current on average, only supercurrent. See Fig. 2. This synchronized behavior will persist as the disorder is increased, provided

$$
\left|\alpha \frac{\left(I_{L}^{2}-I_{R}^{2}\right)}{I_{T, B}^{\min I}}\right| \leqslant 1,
$$

where $\alpha$ is a numerical constant equal to $\frac{3}{16}$. When this condition fails, an abrupt transition to a desynchronized state occurs (Fig. 3). Condition (2), describing the boundary between coherence and incoherence in the plaquette, is determined by the nonresonance condition at $\mathscr{O}\left(\epsilon^{2}\right)$ in the asymptotic expansion, and coincides with the destruction of the frequency-locked state through a degenerate saddle-node bifurcation. (A detailed derivation will appear elsewhere.) ${ }^{13}$

This transition formula strikingly shows that the plaquette can tolerate large amounts of disorder before synchronization is lost (e.g., a 10\% variation in the critical currents is not enough to destroy locking). In Fig. 4 we plot the critical boundary (2) separating the synchronized and desynchronized states, and compare this with the results of numerical simulations. It is clear that the transition criterion (2) remains accurate even for relatively low bias currents, well outside its domain of formal asymptotic validity. We note, however, that the robustness of the plaquette to disorder will

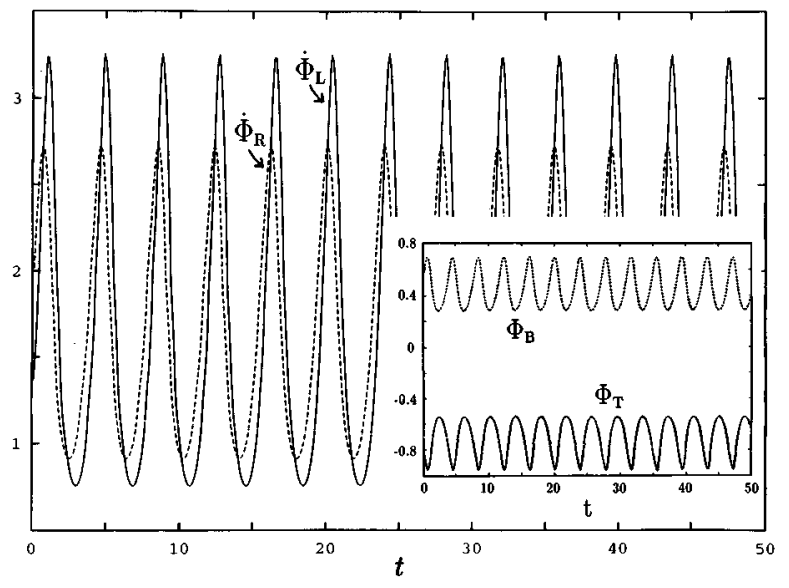

FIG. 2. Synchronized state at $\left(I_{T}=0.2, I_{B}=0.3, I_{L}=1.5, I_{R}=1.0, I\right.$ $=2.0)$. The voltage oscillations of the vertical junctions $\dot{\Phi}_{L}(t), \dot{\Phi}_{R}(t)$ show frequency locking. Note (inset) that phases $\Phi_{T}(t), \Phi_{B}(t)$ oscillate with zero average growth, signifying that no net regular current flows across the horizontal junctions.

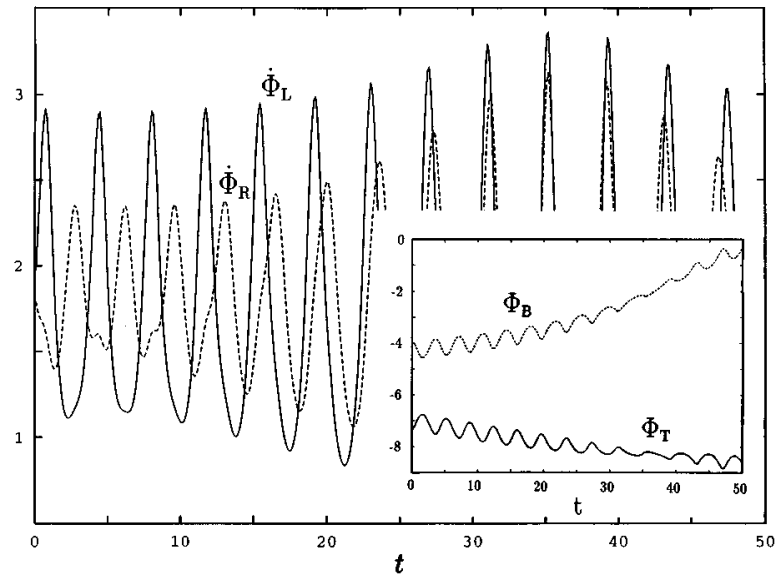

FIG. 3. Desynchronized state at $\left(I_{T}=0.05, I_{B}=0.07, I_{L}=1.5, I_{R}\right.$ $=1.0, I=2.0)$. The vertical voltage oscillations $\dot{\Phi}_{L}(t), \dot{\Phi}_{R}(t)$ are unlocked. The horizontal phases $\Phi_{T}(t), \Phi_{B}(t)$ (inset) display linear growth on average.

not fully carry over to the general case of $N \times M$ arrays. (We will return to this point at the end of this letter.)

The entire synchronization-desynchronization process can be understood from a simple intuitive argument. In the absence of disorder, the in-phase state of the plaquette is stable. Weak disorder will therefore merely deform this periodic solution, not destroy it; consequently, the vertical junctions of the plaquette will remain frequency-locked. Physically, this frequency-locking is accomplished by the horizontal junctions, which shunt net supercurrnet between the vertical junctions and thereby effectively couple them. (Replacing the horizontal junctions by inductors would presumably achieve the same purpose, which indeed seems to

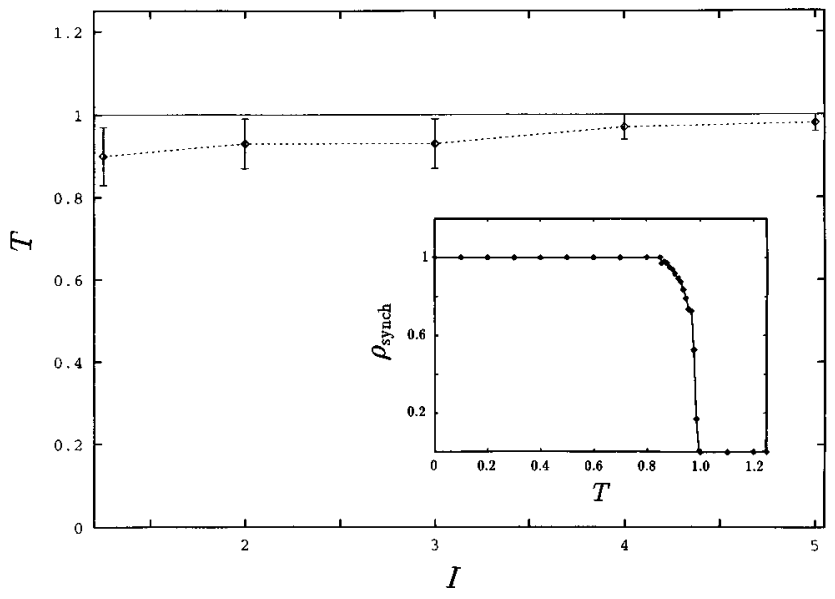

FIG. 4. Transition boundary between frequency-locked and unlocked states. The quantity $T$ denotes the left-hand side of the transition formula (2); the horizontal line at $T=1.0$ defines the theoretical threshold. For different bias currents $I$, the critical currents $I_{R}, I_{T}, I_{B}$ were randomly chosen in the interval $(0,1)$, with $I_{L}$ held fixed at 1.0. A statistical sampling shows that below (respectively, above) the numerically determined curve, most states $(\geqslant 97 \%)$ are synchronized (respectively, unsynchronized). Within the transition regime around the curve (as defined by the error bars), states of both types were found, depending upon the particular sample. The inset shows in more detail the data for $I=2$ : the fraction of states that are synchronized $\left(\rho_{\text {synch }}\right)$ shows a sudden transition near the theoretically predicted threshold. Note that the desynchronized states are only found for very high levels of disorder. 
be the case ${ }^{10}$.) The amount of shunt current required for locking can be estimated as follows: ignoring any ac oscillations, we simply replace the shunt current $\Delta$ in (1a) $-(1 \mathrm{~d})$ by its time average $\langle\Delta\rangle$. The equations may now be integrated directly, and we find that the vertical junctions $\Phi_{L}, \Phi_{R}$ overturn with an average frequency of $\sqrt{(I-\langle\Delta\rangle)^{2}-I_{L}^{2}}$, $\sqrt{(I+\langle\Delta\rangle)^{2}-I_{R}^{2}}$, respectively. Equating these two frequencies yields

$$
\langle\Delta\rangle=\frac{I_{R}^{2}-I_{L}^{2}}{4 I} .
$$

As the disorder in the system is increased, the horizontal junctions will simply shunt the required supercurrent (3), maintaining the frequency-locking. However, if the disorder becomes too large, the required shunt current will exceed the maximum allowable supercurrent through the horizontal junctions, $\left(I_{T}, I_{B}\right)$. At this point, the horizontal junctions will pass regular current in addition to supercurrent, and locking between the vertical junction is destroyed; the plaquette enters a desynchronized state. The critical transition between locked and unlocked behavior occurs when $|\langle\Delta\rangle|$ $=\min \left(I_{T}, I_{B}\right)$. From (3), it follows instantly that the plaquette will be synchronized provided a condition identical to (2) is satisfied, where now $\alpha=1 / 4$. Thus, this simple physical argument effectively reproduces the previous result of the formal asymptotic analysis. The slight difference in the numerical factor $\alpha$ can be attributable to our neglect of the ac-component of the shunt current in this latter physical description. The physical interpretation of the transition criterion (2) is now clear: frequency locking is lost when the average supercurrent through either of the horizontal junction nears its maximum level.

It is interesting to consider a slight variation of the basic plaquette problem: In the preceding analysis, disorder was introduced through variations in the critical currents only. If the effects of variations in the junctions' resistances are also included, a generalized transition formula analogous to (2) can be obtained. ${ }^{13}$ Both types of disorder are found to contribute to the synchronization/desynchronization process, and as before, we find that the plaquette is relatively robust to disorder. The only exception occurs in the regime of very high bias current, in which case small variations in the resistances can destabilize the system. We can also consider the effects of the plaquette's self-magnetic field on its synchronization properties by introducing a modified loop constraint: $\Phi_{T}+\Phi_{R}-\Phi_{B}-\Phi_{L}=-L \Delta$, where $L \Delta$ is the (nondimensionalized) magnetic flux through the plaquette (see, e.g., Refs. 14 and 15). We observe, however, that the earlier physical arguments describing frequency locking in the plaquette remain essentially unaltered, and thus although the exact transition point between locked and unlocked behavior may shift, the overall ability of the plaquette to withstand large amounts of disorder will be preserved. This is borne out by numerical simulations, although a complete analysis for this case has not been carried out.

Finally, from our analysis of the plaquette, we can garner insight into what can occur for larger array systems, which can fruitfully be regarded as a collection of plaquettes. For example, by joining several plaquettes together in a horizon- tal row (with bias current injected vertically), forming a $2 \times M$ array, we expect that the robust frequency-locking identified in the single plaquette will extend across the entire row. Mathematically, this is suggested by observation that the inphase solution of a $2 \times M$ array with zero disorder is stable $^{9}$ (just as in the case of a single plaquette), and thus sufficiently weak disorder will only deform this solution, not destroy its periodicity. The vertical junctions in the row will therefore remain synchronized. Physically, this is accomplished by nonzero supercurrents shunted across the horizontal junctions. Numerical simulations have confirmed this result for a $2 \times 3$ array. If instead we consider a vertical column of plaquettes, forming an $N \times 2$ array (a "ladder"), a quite different effect appears. This stems from the fact that the inphase solution of an $N \times 2$ identical-junction array (with $N>2$ ) is not fully stable, owing to the presence of $N-2$ neutrally stable modes. ${ }^{9,16}$ These neutral modes correspond to perturbing one plaquette in the column with respect to another. Consequently, when (arbitrarily small) disorder is introduced, the periodicity of the inphase state is not expected to be preserved, and the junctions in different rows will operate at different frequencies; the frequency-locking mechanism operating within a single plaquette does not extend vertically downward between different plaquettes. This is consistent with the numerical results of Refs. 6 and 17, and we have rigorously verified this for the case $N=3$ by means of a multiple time scale analysis similar to that described earlier. Hence, we are led to the expectation that in $N \times M$ arrays of RS Josephson junctions with weak disorder, the junctions within a given row will remain frequency-locked, but those in different rows will operate with different frequencies.

We gratefully acknowledge useful discussions with Sam Benz, Peter Booi, and Dick Kautz. This work was supported by the Office of Naval Research.

\footnotetext{
${ }^{1}$ A. K. Jain, K. K. Likharev, J. E. Lukens, and J. E. Sauvageau, Phys. Rep. 109, 309 (1984).

${ }^{2}$ K. K. Likharev, Dynamics of Josephson Junctions and Circuits (Gordon and Breach, Philadelphia, 1986).

${ }^{3}$ R. P. Robertazzi and R. A. Buhrman, IEEE Trans. Mag. 25, 1384 (1989).

${ }^{4}$ D. R. Tilley, Phys. Lett. A 33, 205 (1970).

${ }^{5}$ K. Wan, A. K. Jain, and J. E. Lukens, Appl. Phys. Lett. 54, 1805 (1989); IEEE Trans. Mag. MAG-27, 3339 (1991).

${ }^{6}$ M. Octavio, C. B. Whan, and C. J. Lobb, Appl. Phys. Lett. 60, 766 (1992).

${ }^{7}$ S. P. Benz and C. J. Burroughs, Appl. Phys. Lett. 58, 2162 (1991).

${ }^{8}$ J. Lukens, in Superconducting Devices, edited by S. T. Ruggiero and D. A. Rudman (Academic, San Diego, 1991), p. 105.

${ }^{9}$ K. Wiesenfeld, S. P. Benz, and P. A. A. Booi, J. Appl. Phys. 76, 3835 (1994).

${ }^{10}$ R. L. Kautz, IEEE Transactions on Superconductivity, Proceedings of the Superconductivity Conference, Boston, 1994 (to be published).

${ }^{11}$ By scaling the vertical and horizontal junctions differently, we effectively allow for more variation among the four critical currents $I_{L}, I_{R}, I_{T}, I_{B}$, and thus can describe a more general situation than if we had instead chosen $I_{T}, I_{B} \sim \epsilon$.

${ }^{12}$ A. H. Nayfeh, Introduction to Perturbation Methods (Wiley, New York, 1981).

${ }^{13}$ A. S. Landsberg, Y. Braiman, and K. Wiesenfeld, Phys. Rev. B (to be published).

${ }^{14}$ J. R. Phillips, H. S. J. van der Zant, J. White, and T. P. Orlando, Phys. Rev. B 47, 5219 (1993).

${ }^{15}$ R. D. Bock, Phys. Rev. B 49, 10009 (1994).

${ }^{16}$ P. Hadley Ph.D. thesis, Stanford University (1989).

${ }^{17}$ C. B. Whan et al., Bull. Am. Phys. Soc. 40, 117 (1995).
} 